\title{
Function and Effects of L-cysteine on the Speciation Analysis of Mercury by High Performance Liquid Chromatography Coupled with On-line Cold Vapor Generation Atomic Fluorescence Spectrometry
}

\author{
Xue-jiao NAN \\ Tianjin Key Lab of Marine Resources \& Chemistry, \\ College of Chemical Engineering and Materials Science, \\ Tianjin University of Science \& Technology, \\ Tianjin, 300457, PRC
}

\author{
Xiao-ping $\mathrm{YU}^{*}$ \\ Tianjin Key Lab of Marine Resources \& Chemistry, \\ College of Chemical Engineering and Materials Science, \\ Tianjin University of Science \& Technology, \\ Tianjin, 300457, PRC \\ College of Chemistry and Materials Science, \\ Northwest University, Xi'an, 710127, PRC \\ e-mail: yuxiaoping@tust.edu.cn
}

\section{Ya-fei GUO}

Tianjin Key Lab of Marine Resources \& Chemistry, College of Chemical Engineering and Materials Science, Tianjin University of Science \& Technology, Tianjin, 300457, PRC

College of Chemistry and Materials Science, Northwest University, Xi'an, 710127, PRC

\begin{abstract}
To study the function and effects of L-cysteine on the separation analysis of inorganic divalent mercury [ $\mathrm{Hg}(\mathrm{II})]$, methylmercury (MeHg) and ethylmercury (EtHg), the separation, transformation and signal intensity of mercury speciation were investigated with liquid chromatography coupled on-line cold vapor generation atomic fluorescence spectrometry (LC-CV-AFS) and CV-AFS systematicly. The results show that $L$-cysteine in mobile phase has no significant effect on the signal intensity of organic mercury and doesn't lead to speciation transformation of organic mercury. However, L-cysteine has decisive effect on separation of different mercury speciation. Therefore, results show that L-cysteine only performed a complexing agent in mobile phase to separate mercury speciation.
\end{abstract}

Keywords- Mercury; Speciation analysis; LC-CV-AFS; Lcysteine.

\section{INTRODUCTION}

Mercury $(\mathrm{Hg})$ is one of the most hazardous pollutants, the toxicity, bioavailability and biochemical behaviors of mercury are not only dependent on its total concentration but on its chemical forms [1]. Hence, mercury speciation analysis is significant to assess mercury toxicity and better understand the mercury biogeochemical cycles.

LC-CV-AFS is widely accepted for mercury speciation analysis due to wide linear range, high sensitivity, simplicity, excellent detection limits etc [2-3]. Literatures have reported that organic mercury is usually necessary to be converted into inorganic divalent mercury before mercury vapor generation in AFS. The common method is on-line conversion by post-column oxidation using strong oxidant

\section{Tian-long DENG}

Tianjin Key Lab of Marine Resources \& Chemistry, College of Chemical Engineering and Materials Science,

Tianjin University of Science \& Technology,

Tianjin, 300457, PRC

e-mail: tldeng@ tust.edu.cn

with UV irradiation [4-5] and microwave radiation [6-7]. However, it was found when mobile phase contains Lcysteine, no strong oxidant and post-column processing such as ultraviolet or microwave are needed [8]. However, the reasons for L-cysteine eliminating the pretreatment of postcolumn oxidation are still unknown or unclear.

In this work, we designed several experiments to investigate the function and effects of L-cysteine on mercury speciation analysis by LC-CV-AFS.

\section{EXPERIMENTAL}

\section{A. Instruments}

$\mathrm{Hg}(\mathrm{II}), \mathrm{MeHg}$ and $\mathrm{EtHg}$ were carried out by a Agela MPC18 chromatographic column $(150 \mathrm{~m} \times 4.6 \mathrm{~mm}$ i.d., $5 \mu \mathrm{m})$ protected by a guard column. A $7725 \mathrm{i}$ injection valve with $100.0 \mu \mathrm{L}$ sample loop (Rheodyne, Japan) was used for sample introduction. Mobile phase ( $8 \mathrm{mM}$ L-Cys $+60 \mathrm{mM}$ ammonium acetate) was delivered by a LC-10AT liquid pump (Shimadzu, Japan), and delivered into a SA-10 speciation analysis pretreatment device (Titan, China) for cold vapor generation. A peristaltic pump was used for the introduction of oxidant (2.0\% K2S2O8), air, current-carrying $(6.0 \% \mathrm{HCl})$ and reductant $(2.0 \% \mathrm{KBH} 4)$. Finally, the mercury vapor generated was analyzed by an atomic fluorescence spectrometer (Titian, China).

\section{B. Standard Solutions and Reagents}

All reagents used were guaranteed reagent, and solutions were prepared using double deionized water (DDW) with resistivity no less than $18 \mathrm{~m} \Omega \bullet \mathrm{cm}$. $\mathrm{Hg}(\mathrm{II}), \mathrm{MeHg}, \mathrm{EtHg}$ stock 
solutions were obtained from National Standard Substance Center in China.

\section{RESULTS AND DISCUSSION}

\section{A. Effects of L-cysteine on the Separation and Speciation Transformation of Organic Mercury}

Wang et al [8] thought that L-cysteine could weaken the $\mathrm{C}-\mathrm{Hg}$ bond in organic mercury by forming complexes, which was broken in the presence of $\mathrm{BH} 4-$ and $\mathrm{HCl}$. Finally, organic mercury was reduced to elemental mercury in AFS. However, no study has verified this hypothesis or found out its exact mechanism. Given this, several experiments were designed to investigate probable reasons.

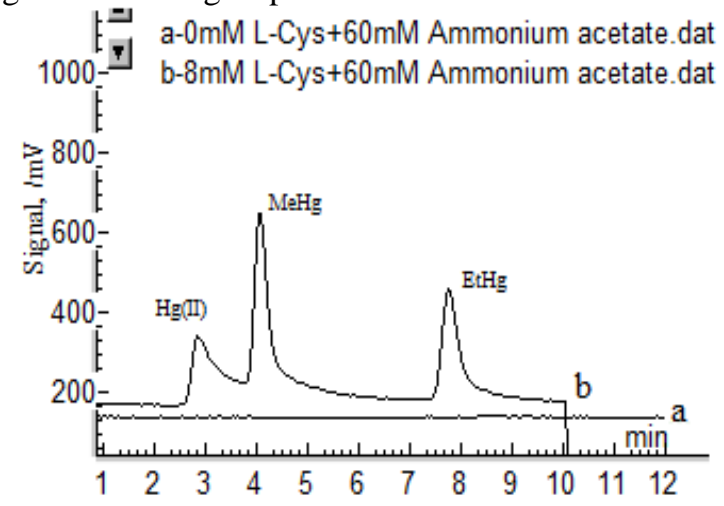

Figure 1. Effect of L-cysteine on the separation of mercury species by reverse-phase chromatography.

In our works, when no L-cysteine was added into the mobile phase, no any chromatographic peaks were presented in the chromatogram (Figure 1). This means L-cysteine is essential for the separation of mercury species. In fact, Lcysteine is usually added into mobile phase as a complexing reagent to form hydrophilic chelates with mercury, by which the completely baseline separation of mercury species is achieved by C18 column [9-10]. If L-cysteine can alone convert from organic mercury to inorganic form, the chromatogram of $\mathrm{MeHg}$ dissolved in L-cysteine solution should presence a new chromatographic peak and decrease or disappear of $\mathrm{MeHg}$ chromatographic peak. However, even though there existed a small chromatographic peak at the retention time of $\mathrm{Hg}$ (II) (Figure 2), it resulted from the impurity of L-cysteine reagent compared with single Lcysteine solution. Meanwhile, we found that the signal intensity of $\mathrm{MeHg}$ both in L-cysteine and DDW solution was basically equal. These results suggested that the addition of L-cysteine in $\mathrm{MeHg}$ solution didn't lead to the conversion from organic mercury to inorganic form.

L-cysteine in acidic medium is usually used as a reductant to reduce $\mathrm{Se}(\mathrm{VI})$ or $\mathrm{As}(\mathrm{V})$ into $\mathrm{Se}(\mathrm{IV})$ or $\mathrm{As}(\mathrm{III})$ during the total amount analysis by HG-AFS [11-12]. Therefore, we further investigate the effects of L-cysteine in acidic medium on $\mathrm{MeHg}$ signal. Two samples containing 20 $\mu \mathrm{g} / \mathrm{L} \mathrm{MeHg}+6 \% \mathrm{HCl}$ were prepared and one of them was adjusted to obtain $8 \mathrm{mM}$ L-cysteine solution. There presented an obvious $\mathrm{Hg}$ (II) peak both in the chromatograms of $\mathrm{MeHg}+\mathrm{HCl}$ and $\mathrm{MeHg}+\mathrm{L}$-cysteine +
$\mathrm{HCl}$ samples (Figure 3). We found that the signal intensity of $\mathrm{MeHg}$ in both of them was almost the same, which suggested that L-cysteine in $\mathrm{MeHg}$ solution didn't cause the conversion from organic mercury to inorganic form even under acidic condition.

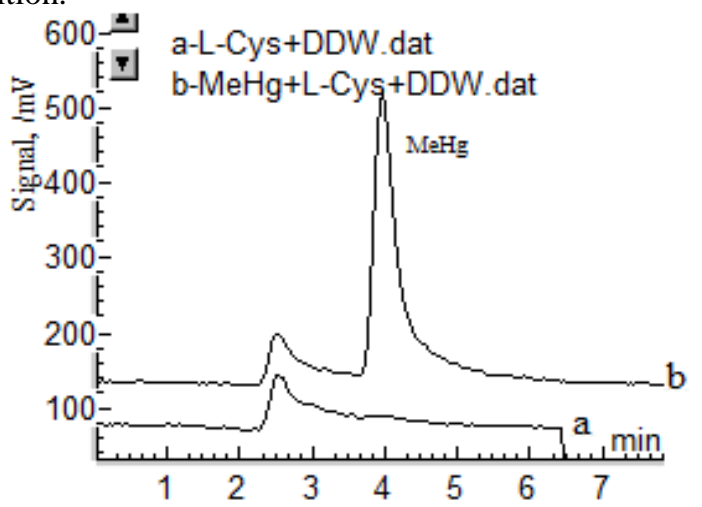

Figure 2. Effect of L-cysteine on the signal intensity of $\mathrm{MeHg}$.

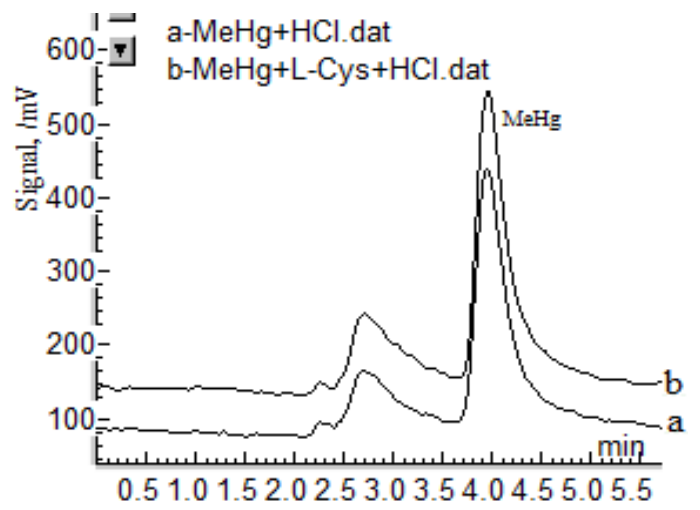

(a)

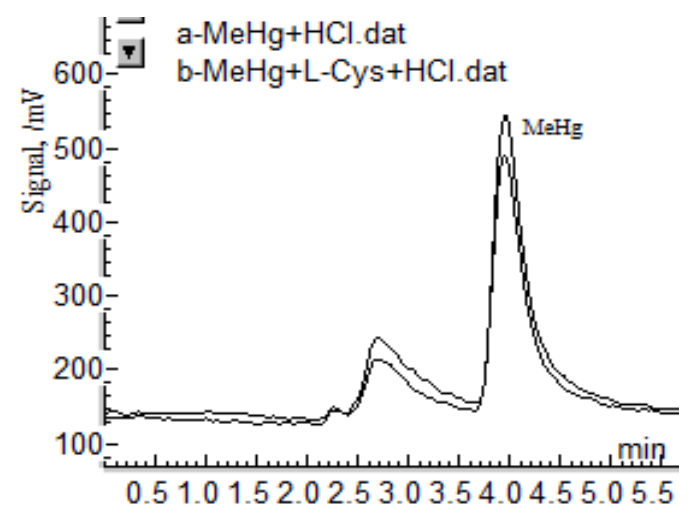

(b)

Figure 3. Effect of L-cysteine in acid medium on the signal intensity of MeHg.

Based on the results, it seems that L-cysteine doesn't lead to speciation transformation or the enhancement of fluorescence signal. However, it is strange that why organic 
mercury species can produce obvious fluorescence signals in CV-AFS, that is to say why organic mercury can generate mercury atomic vapor without post-column oxidation or UV irradiation operation. Is it really the case that "L-cysteine can weaken the $\mathrm{C}-\mathrm{Hg}$ bond in organic mercury by forming complexes, which was broken in the presence of $\mathrm{BH} 4$ - and $\mathrm{HCl}$ to generate elemental mercury"? In order to verify this hypothesis, further experiments were carried out.

\section{B. Effects of L-cysteine on the Signal Intensity of OrganicMercury}

$\mathrm{MeHg}$ was prepared in DDW, $8 \mathrm{mM}$ L-cysteine and $6 \%$ $\mathrm{HCl}+8 \mathrm{mM}$ L-cysteine to obtain $20 \mu \mathrm{g} / \mathrm{L} \mathrm{MeHg}$ solutions, respectively. Three other reduplicative samples were prepared and exposed under UV irradiation about $10 \mathrm{~min}$ before analysis by CV-AFS. The results were shown in the form of histogram in Figure 4.

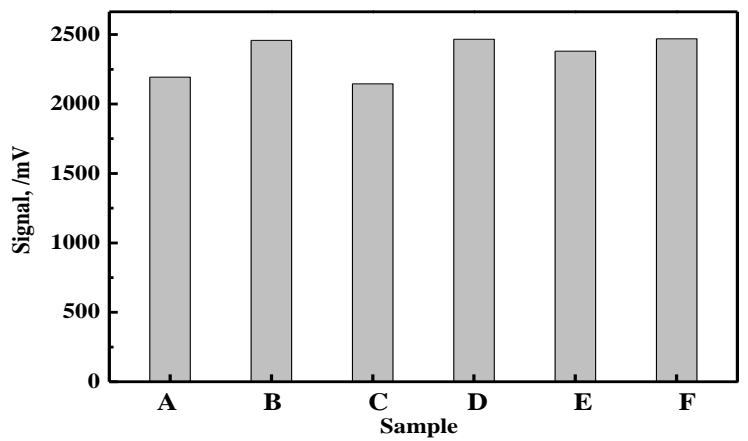

A: MeHg+DDW (non-UV); B: MeHg+DDW (UV); C: MeHg+L-cysteine (non-UV); D: MeHg+ L-cysteine (UV);

E: $\mathrm{MeHg}+\mathrm{L}-$ cysteine+HCl (non-UV); $\quad \mathrm{F}: \mathrm{MeHg}+\mathrm{L}-$ cysteine+HCl (UV).

Figure 4. Determination of $\mathrm{MeHg}$ in different mediums and at different conditions by CV-AFS.

If $\mathrm{L}$-cysteine weakens the $\mathrm{C}-\mathrm{Hg}$ bond in organic mercury and finally leads to the generation of mercury atomic by reacting with $\mathrm{BH} 4-$ and $\mathrm{HCl}, \mathrm{MeHg}$ signal in L-cysteine solution will be stronger than that in DDW. However, compared the height between bar A and C (Figure 4), we found that $\mathrm{MeHg}$ signal in the solution with or without Lcysteine was basically similar under no UV irradiation. Even though $\mathrm{MeHg}$ signal in L-cysteine $+\mathrm{HCl}$ medium (bar E) was slightly higher than that both in DDW and L-cysteine solution, the enhancement of signal mainly resulted from the effect of $\mathrm{HCl}$ as the increase of acidity strengthens the reducibility of BH4-. Meanwhile, even the samples were offline irradiated by UV light before analysis, there hadn't obvious signal difference of $\mathrm{MeHg}$ in DDW and L-cysteine medium compared B with D in Figure 4. It is interesting that when the samples were irradiated by UV light, $\mathrm{MeHg}$ signal both in DDW and L-cysteine was distinct higher by comparison between bar $\mathrm{A}$ and $\mathrm{B}$ as well as bar C and D. However, note that no new peaks were presented in the chromatograms of $\mathrm{MeHg}$ prepared in DDW or L-cysteine after off-line UV irradiation. That means the increase of $\mathrm{MeHg}$ signal doesn't result from the conversion of mercury speciation through the UV irradiation. It is speculated that organic mercury in the ground state is excited to a high energy state under the UV irradiation, by which it contributes to the reduction of organic mercury by BH4-, therefore, higher signals are finally obtained after UV irradiation.

\section{CONCLUSIONS}

$\mathrm{Hg}$ (II), MeHg and EtHg can be well sepatated by $\mathrm{C} 18$ column, because the sulfydryl in L-cysteine can bond with mercury atomic in mercury compounds to form hydrophilic chelates. However, the complexation between L-cysteine and mercury compounds doesn't cause generation of organic mercury flourescence signals, which is consistent with the resluts that the signals of $\mathrm{MeHg}$ both in DDW and Lcysteine medium are basically the same. That means Lcysteine has no effect on the signal intensity and speciation transformation of organic mercury. In conclusion, L-cysteine only acts as a complexing agent in mobile phase to separate mercury speciation.

\section{REFERENCES}

[1] H.H. Harris, I.J. Pickering and G.N. George, The chemical form of mercury in fish, Sci., 301, 29, 1203 (2003).

[2] R. Falter, H. Hintelmann and P. Quevauviller, Conclusion of the workshop on sources of error in methylmercury determination during sample preparation, derivatisation and detection, Chemosphere, 39, 7, 1039 (1999).

[3] P. Houserova, D. Matejicek, V. Kuban, et al, Liquid chromatographic-cold vapour atomic fluorescence spectrometric determination of mercury species, J. Sep. Sci., 29, 2, 248 (2006).

[4] D.P.C.D. Quadros, B. Campanella, M. Onor, et al., Mercury speciation by high-performance liquid chromatography atomic fluorescence spectrometry using an integrated microwave/UV interface. Optimization of a single step procedure for the simultaneous photo-oxidation of mercury species and photogeneration of $\mathrm{Hg}^{0}$, Spectrochim. Acta B, 101, 312 (2014).

[5] G. Acostaa, A. Spissoa, L.P. Ernandeza, et al, Determination of thimerosal in pharmaceutical industry effluents and river waters by HPLC coupled to atomic fluorescence spectrometry through postcolumn UV-assisted vapor generation, J. Pharmaceu.l Biomed., 106, 79 (2015).

[6] B. Campanella, M. Onor, M. C. Mascherpa, et al, Determination of thiomersal by flow injection coupled with microwave-assisted photochemical online oxidative decomposition of organic mercury and cold vapor atomic fluorescence spectroscopy, Anal. Chim. Acta, 804, 66 (2013).

[7] Y. Wu, Y. Lee, L. Wu and X. D. Hou, Simple mercury speciation analysis by CVG-ICP-MS following TMAH pre-treatment and microwave-assisted digestion, Microchem. J., 103, 1, 105 (2012).

[8] Z.H. Wang, Y.G. Yin, B. He, et al., L-cysteine-induced degradation of organic mercury as a novel interface in the HPLC-CV-AFS hyphenated system for speciation of mercury, J. Anal. Atom. Spectrom., 25, 6, 749 (2010).

[9] G.M. Guang, Y.M. Guo, X.C. Chen, et al, HPLC-AFS determination of species of mercury in aquatic products, Phy. Testing Chem. Anal. B, 49, 7, 840 (2013).

[10] X.Y. Jia, Y. Han, X.L. Liu, et al, Speciation of mercury in water samples by dispersive liquid-liquid microextraction combined with high performance liquid chromatography-inductively coupled plasma mass spectrometry, Spectrochim. Acta B, 66, 1, 88 (2011).

[11] S.J. Chen and Y.J. Wang, Determination of total inorganic arsenic in meat and meat products in L-cysteine as reduction, Modern Instrument, 6, 66 (2008). (in chinese) 
[12] S.H. Zou, Determination of arsenic in water by hydride generationatomic fluorescence spectrometry with L-cysteine as Pre-reduction
Agent, Water Technol., 3, 1, 52 (2009). (in chinese) 